\title{
A study on performance of planing-wing hybrid craft
}

\author{
Hamid Zeraatgar, Assistant Professor \\ Ali Bakhshandeh Rostami, MSc. in Naval Architecture \\ Abolfazl Nazari, MSc. in Naval Architecture \\ Amirkabir University of Technology, Tehran, Iran
}

\begin{abstract}
A hybrid craft is defined as a combination of different lifting surfaces. The lift force can be of aerodynamic or hydrodynamic character. This research deals with the conditions of using planing mono-hulls equipped with a couple of wings, called planing-wing hybrid craft. A parametric study is conducted by using Vortex Lattice Method to determine feasibility of using an aerodynamic wing mounted to planing craft. It has been determined that the wing area should be more than twice as much as the wetted surface of the craft, to get an effective wing for planing craft. Furthermore, the wing should be so designed as to obtain its high aspect ratio. Maximum lift force contribution resulting from different wing configurations considered in this study is less than 35 percent of the total hydrodynamic lift force at an arbitrary condition. On this basis it may be concluded that the wing may be effectively used only in special conditions, hence this is not recommended for general application.
\end{abstract}

Key words: hydrodynamics; ground effect; wing; planing; aerodynamics; VLM

\section{INTRODUCTION}

People are always looking for increasing productivity and improving performance of transportation. Serious limitations on increasing speed and gradual development of existing marine crafts led to a new generation of craft, known as hybrid crafts. The objective of this paper is to examine performance of one kind of such crafts, namely the planing-wing hybrid craft in which hydrodynamic and aerodynamic lift forces mainly supports the craft's weight at service speed.

Due to higher density and viscosity of water than air, large speed of a body in water causes its frictional and wave-making resistance greater than in case of moving in air. One solution to reduce this type of resistance is reducing the wetted surface of craft. Although lifting surfaces produce a higher lift force and reduce craft's draught but they may cause also some detrimental effects such as cavitation of hydrofoils, degradation of craft's performance in waves, reduction of its stability etc. The restrictions may put obstacles on increasing the craft speed.

WIG crafts (Wing- In- Ground- effect crafts) are the crafts which make use of aerodynamic forces at service speed. Undoubtedly, the use of aerodynamic forces can be considered as a worthy contribution to reaching higher speeds in marine transportation. The reason is that a significant lift force can act onto a craft moving near water surface, by which WIG crafts are able to achieve very high speeds. However, the WIG crafts are facing some problems such as structural and dynamic complexities, construction cost and safety [1] as well as low efficiency of air propulsion system. Another disadvantage of WIG craft is inability to perform even very simple manoeuvres in planing mode.
Some types of high speed crafts, so called the hybrid crafts, may use hydrodynamic lift of planing hull, hydrodynamic lift of hydrofoils and aerodynamic lift of aerofoils. It means that in the hybrid craft the capability of various lifting surfaces are used to gain more efficiency and performance quality.

This study is intended for examining usage of aerodynamic forces including ground effect and hydrodynamic forces simultaneously to increase the efficiency and performance quality. A high lift-to-drag ratio of the aerodynamic foils can make a fundamental change in power consumption of propulsion system and allow reaching higher speeds.

The main question is what should be the wing dimensions so that a significant part of the craft weight to be supported by air lift? Is there any advantage from using air lift instead of hydrodynamic lift?

In this context a literature review shows that there are some products which use a combination of different kinds of lift force, and that none of them have been theoretically discussed. In some cases only patent registration was done. In 1976, Shipps [2] introduced a type of hybrid craft, known as "tunnel hull", used as a race boat. This design in which two planing hulls were applied at the end of a ram wing and considered as end plates, could promptly demonstrate its better performance than planing mono-hulls; and thus a major step was made in designing the race crafts. One of the benefits of this design was the use of aerodynamic lift force for bearing craft weight. In 1978, Ward [3] presented some results of testing the vessel KUDU II (a modified form of the vessel KUDU I designed in 1976), which could sail at speed up to 78 knot. In 1996, Privalov and Kirillovikh [4] designed a hybrid craft called TAP (Transport Amphibious Platform) which consisted of 
two catamaran- like bodies, an aircraft fuselage, aerodynamic wing and tail. Its motion in close proximity to water surface or ground resulted in forming an air cushion between the hulls. Among other merits it allows to develop a very high speed (250 $\mathrm{km} / \mathrm{h}$ ). In 1997, an ekranocat vessel was presented by Doctor [5] during WIG's97 Conference, in which an aerodynamic superstructure was arranged on catamaran body, resulting in drag reduction by 50 percent, according to the calculations and measurements.

Furthermore, several patents for the use of aerodynamic elements in high speed crafts have been registered in the US [6], and in all of them utilization of ground effect has been indicated as the reason for using the wings. In 2005, a research program was started at Cranfield University, UK, whose goal was the use of aerodynamic forces for high speed craft [7].

Balancing the different forces which act on hybrid crafts has been a target of some research projects in recent years. Collu et al. [8] and [9] has considered longitudinal stability of hybrid crafts in both static and dynamic states.

The motivation for this study is based on the attractive parameters of a boat called Seaphantom, claimed by its manufacturer [10]. The Seaphantom is an innovative hybrid craft whose original idea formed in 2000 was registered as a US patent [6]. In 2004 was built its prototype on which a set of tests and trials was performed. The second unit was built in 2007 and tested in January 2008. The craft's designer and manufacturer tried to use different lifting elements to achieve the maximum possible speed by minimum power. According to the manufacturer's claim, the 300-hp Seaphantom is able to move with the speed of 70 knot which is considered an excellent feature of the boat. Its wings have a low breadth compared to hull breadth, which is other key point to be mentioned about the craft. Tab. 1 shows the Seaphantom specification.

Tab. 1. Seaphantom specification [10]

\begin{tabular}{|c|c|c|}
\hline Item & Quantity & Unit \\
\hline Length overall & 9.27 & $\mathrm{~m}$ \\
\hline Breadth overall & 3.818 & $\mathrm{~m}$ \\
\hline Speed(max) & 70 & $\mathrm{kn}$ \\
\hline Power & 224 & $\mathrm{~kW}$ \\
\hline Engine type & \multicolumn{2}{|c|}{ Mercury race } \\
\hline Engine configuration & \multicolumn{2}{|c|}{ Outboard } \\
\hline Engine speed & 9000 & $\mathrm{rpm}$ \\
\hline
\end{tabular}

The purpose of this study is to examine hydrodynamic advantages of a hybrid craft which uses both the hydrodynamic lift force of a planing body and the aerodynamic lift force resulting from air wings which are placed close to the water level to benefit from aerodynamic lift due to ground effect.

To achieve the above mentioned objective, a model planing-wing hybrid craft on which different air wings are assembled, is selected. A schematic image of the hybrid craft is shown in Fig. 1. The lift and drag forces produced by main body, wings and ground effect are calculated with the use of Vortex-lattice method. Comparison between the lift-to-drag ratio of wings and planing body is done. The total lift force of the aerodynamic wings with respect to craft weight is determined. Finally, the idea of using the planing-wing hybrid craft is discussed and the conditions in which aerodynamic lift force of wing is beneficial for planing mono-hull crafts are formulated.

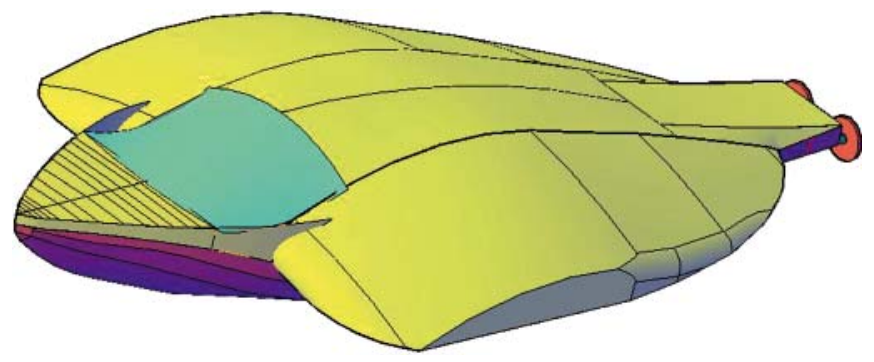

Fig. 1. A schematic image of the model planing-wing hybrid craft

\section{THE PROBLEM STATEMENT}

This research, based on considering the issue of hybrid craft as a combination of aerodynamic and hydrodynamic forces and taking into account the Seaphantom as a model, is aimed at examination of usefulness of this kind of craft. The problem is defined as follows: a planing craft equipped with two wings is designed as a hybrid craft moving in water with the speed in the range of planing mode. The air is blown under the wings due to craft's speed thus the aerodynamic lift is generated. A small gap between wings and water surface results a higher aerodynamic lift due to ground effect. Some further assumptions are as follows:

- The craft has to remain in contact with water.

- Size and geometry of the main hull follow the principles of planing body.

- Dimensions and size of aerodynamic wings are in consistency with general arrangement of a planing craft

- Trim angle of planing body and angle of attack of wing are selected rationally.

- It should be noted that around wings water spray occurs that is not considered here.

- In this study craft steady motion at constant speed in calm water is considered.

- The Vortex-lattice method is used as a tool for both hydrodynamic and aerodynamic force calculations.

- Friction forces are calculated by a regression formula and added to Vortex-lattice method calculation results.

- Gap between water surface and the lowest point of wing is related to wing chord at root section, and marked $\mathrm{H} / \mathrm{C}$, where $\mathrm{H}$ is gap height and $\mathrm{C}$ is chord length of wing root section.

\section{THE GOVERNING EOUATION AND COMPUTER SOFTWARE}

\section{Governing equation}

The Vortex-lattice methods are based on solutions of Laplace's equation. They were first formulated in the late 30s and first called "Vortex Lattice" by Faulkner in 1943. The velocity potential should satisfy Laplace's equation as follows:

$$
\nabla^{2} \Phi=0
$$

where: $\Phi$ is total velocity potential and, for a steady flow, written as follows:

$$
\Phi=\mathrm{Ux}+\phi
$$

where: $\mathbf{U}$ is the uniform stream velocity and $\phi$ is the perturbation potential. Both $\Phi$ and $\phi$ satisfy Laplace's equation. 


\section{Computer software}

To perform aerodynamic calculations of a ground-effect craft a computer software called Autowing based on the boundary element method (called VLM), has been developed by Korneev [11]. In the computer program the main hull and wings are meshed by square panels on which singularities are distributed. This software has been developed specially to design wings for ground-effect crafts. The Autowing is capable of calculating hydrodynamic and aerodynamic lift and drag. The frictional drag could not be calculated by means of the Vortex-lattice method, hence it is computed by using an empirical formula inserted in the Autowing.[11].

\section{THE PROCESS OF RESEARCH}

A planing body whose geometric characteristics are presented in Tab. 2, is selected as a hull. Its dead-rise angle varies from 10 degrees aft to 15 degrees amid-ship. The body lines of the craft and its 3D image are shown in Fig. 2 and 3, respectively. White area in Fig. 3 distinguishes the underwater part of the hull on even keel condition.

Tab. 2. Main particulars of the hybrid craft

\begin{tabular}{|c|c|c|}
\hline Particulars & Quantity & Unit \\
\hline LOA & 18.2 & $\mathrm{~m}$ \\
\hline LBP & 17 & $\mathrm{~m}$ \\
\hline B(max $)$ & 5.6 & $\mathrm{~m}$ \\
\hline Draught & 1.36 & $\mathrm{~m}$ \\
\hline Height & 2.9 & $\mathrm{~m}$ \\
\hline Displacement & 66.57 & $\mathrm{t}$ \\
\hline LCG from aft & 7.957 & $\mathrm{~m}$ \\
\hline
\end{tabular}

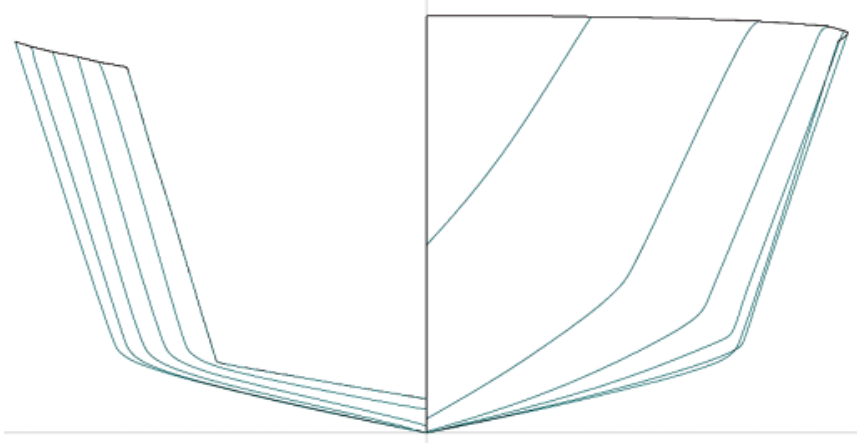

Fig. 2. Body lines of the hybrid craft

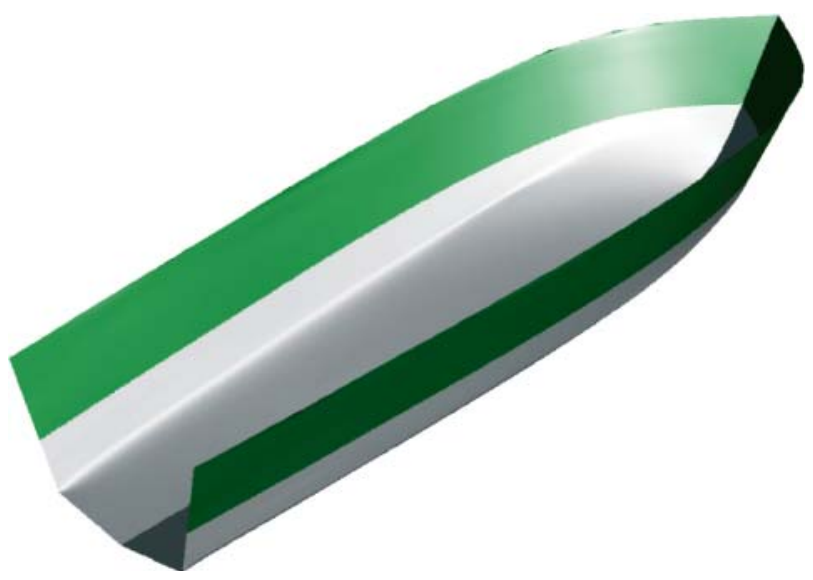

Fig. 3. 3D image of the hybrid craft
The 3D model was used to perform hydrostatic calculations and consequently to obtain centre of buoyancy and displacement of the craft. Furthermore, its wetted surface was calculated at different trim angles and draughts. To determine hydrodynamic lift force the vessel was modelled by using the Autowing, as shown in Fig. 4. The calculations were done for four draughts and five trim angles at six different speeds as given in Tab. 3 .
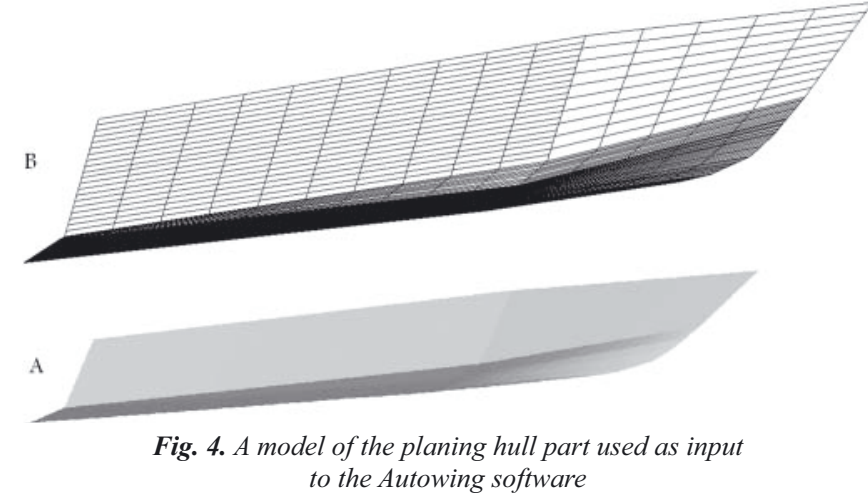

Tab. 3. Input parameters of example calculations

\begin{tabular}{|c|c|c|}
\hline Item & Quantity & Unit \\
\hline Draught & $0.5,0.65,0.8,0.95$ & $\mathrm{~m}$ \\
\hline Trim & $2,3,4,5,6$ & $\mathrm{deg}$ \\
\hline Speed & $25,40,55,70,85,100$ & $\mathrm{kn}$ \\
\hline
\end{tabular}

According to the research on the ground effect [12], the pressure side of wing surface, which has been carefully examined in the case study, should be flat. The wing shape is like an inverse delta, known as Lippisch type, whose important advantage is providing the craft with a better stability [13].

The aerodynamic calculations were also done by using the Autowing. The wing geometry is shown in Fig. 5. In Fig. 6 a typical airfoil section of the wing is shown. The dimensions of the wings are selected proportionally to the dimensions of the craft. Tab. 4 shows the ratios of wing dimensions in respect with the craft, where $\mathrm{C}$ and $\mathrm{S}$ are the chord length and span of the wing, respectively. Furthermore, $\mathrm{H}$ is gap height, and $L$ and $B$ are the craft length and beam, respectively (see Fig. 7).

Tab. 4. Wing main dimensions

\begin{tabular}{|c|c|}
\hline Item & Quantity \\
\hline $\mathrm{C} / \mathrm{L}$ & $0.5,0.75,0.9$ \\
\hline $\mathrm{S} / \mathrm{B}$ & $0.5,1,1.5$ \\
\hline $\mathrm{H} / \mathrm{C}$ & $0.05,0.059,0.088$ \\
\hline $\mathrm{C}(\mathrm{m})$ & $8.5,12.75,15.3$ \\
\hline
\end{tabular}

For aerodynamic analysis the gap of $75 \mathrm{~cm}$ measured from water level to the end point of the wing, is considered because, the maximum ground effect happens at a height of less than 10 percent to the wing chord length [14]. To obtain better performance and achieve higher lift force the wings with the initial angle of attack of 7 degrees, are assumed. It means that if the craft is on even keel in water, its wing will have the angle of attack of 7 degrees. 


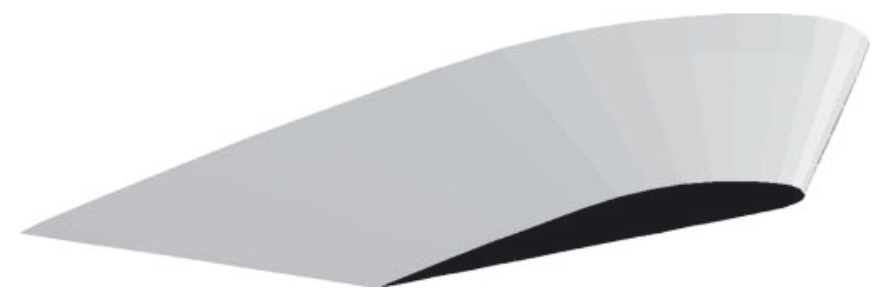

Fig. 5. Wing geometry modelled by the Autowing

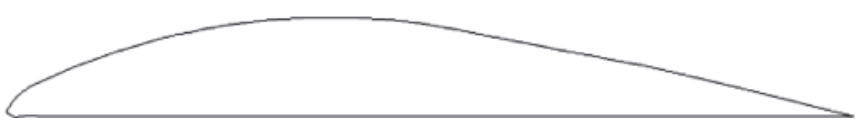

Fig. 6. Typical airfoil section used for wing

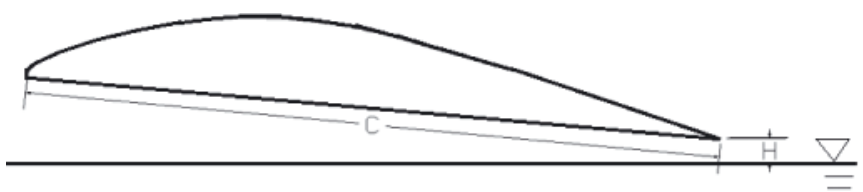

Fig. 7. Parameters defined for ground effect

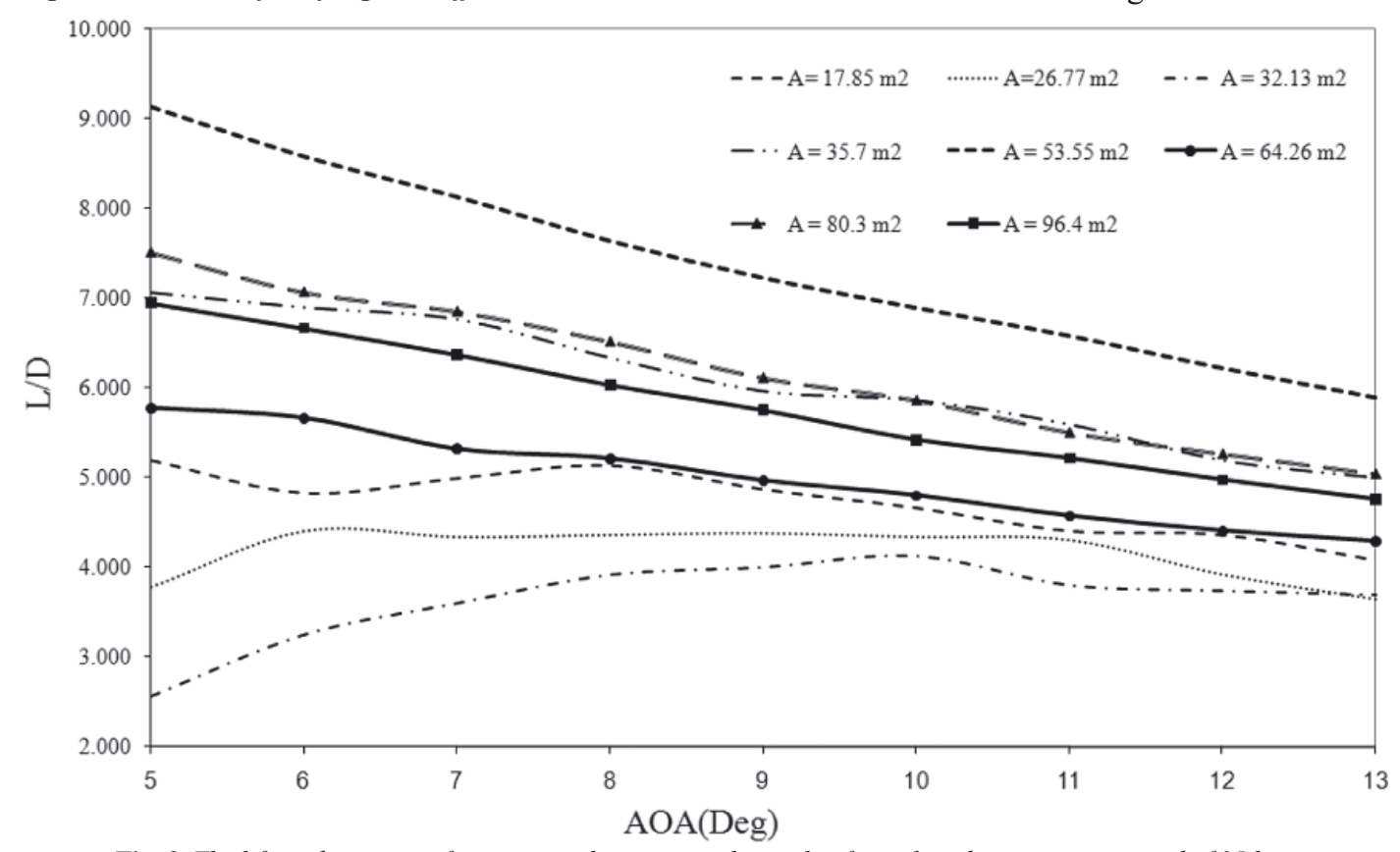

Fig. 8. The lift-to-drag ratio of air wing with respect to the angle of attack and wing area at speed of $25 \mathrm{knot}$

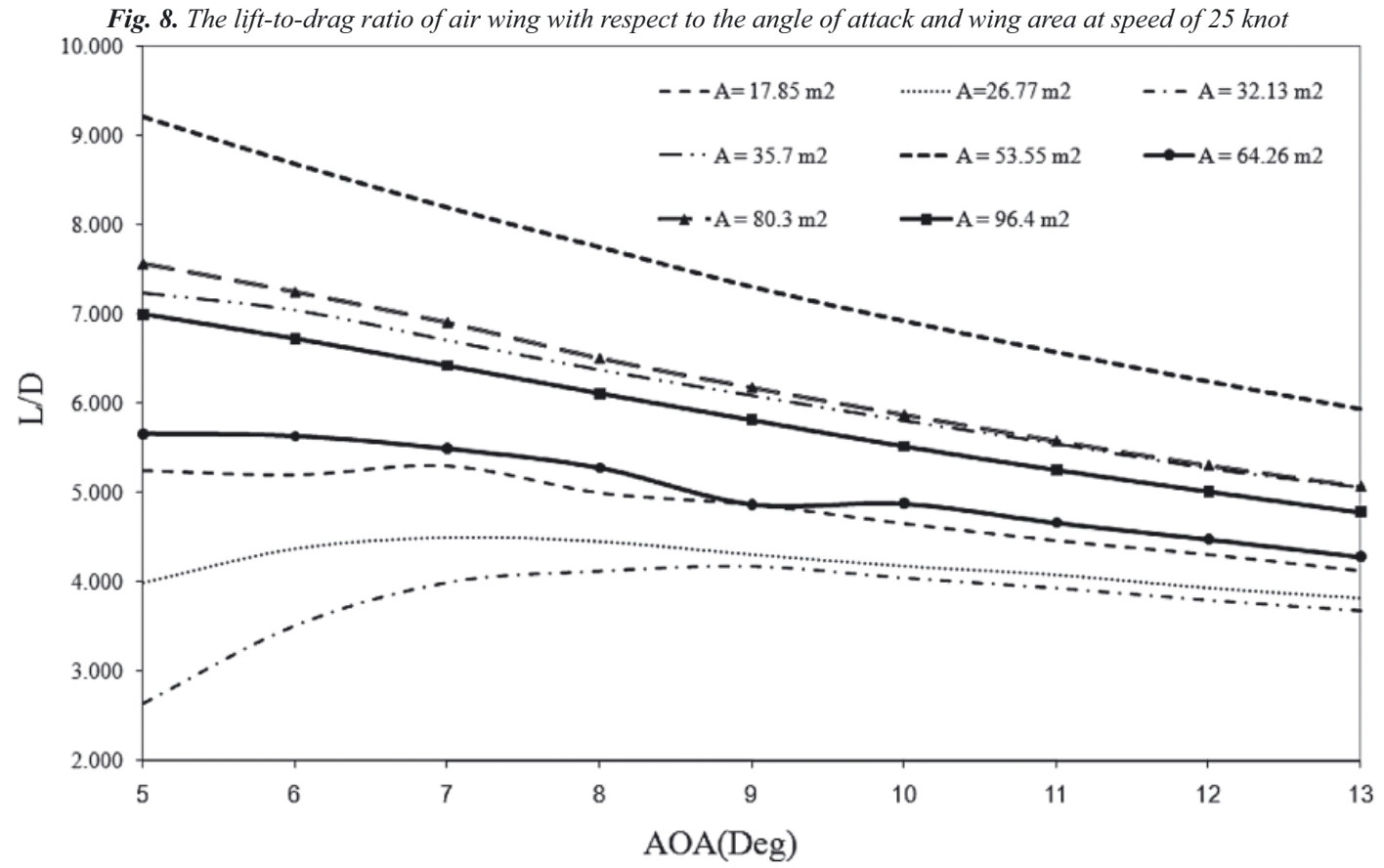

Fig. 9. The lift-to-drag ratio of air wing with respect to the angle of attack and wing area at speed of $55 \mathrm{knot}$
A set of calculations was done for several configurations of the hybrid craft, in which its draught, trim, wing size and speed were systematically changed. Their results are below presented and discussed.

\section{Comparison of the hydrodynamic and aerodynamic lift-to-drag ratios}

Since two elements support craft's weight by dynamic lift, the lift-to-drag ratio is a good measure of merit for comparing different lifting surfaces. Obviously, the higher lift-to-drag ratio the better performance of a lifting surface.

In Fig. 8 through 10, the lift-to-drag ratios are presented in function of angle of attack and wing area for three different speeds. The speeds of 25, 55 and 100 knot were selected to cover the ranges of low, intermediate and very high speeds. It is observed that the lift-to-drag ratio of wing varies from 2.5 to 9.5 and decreases as the angle of attack increases. The ratio 


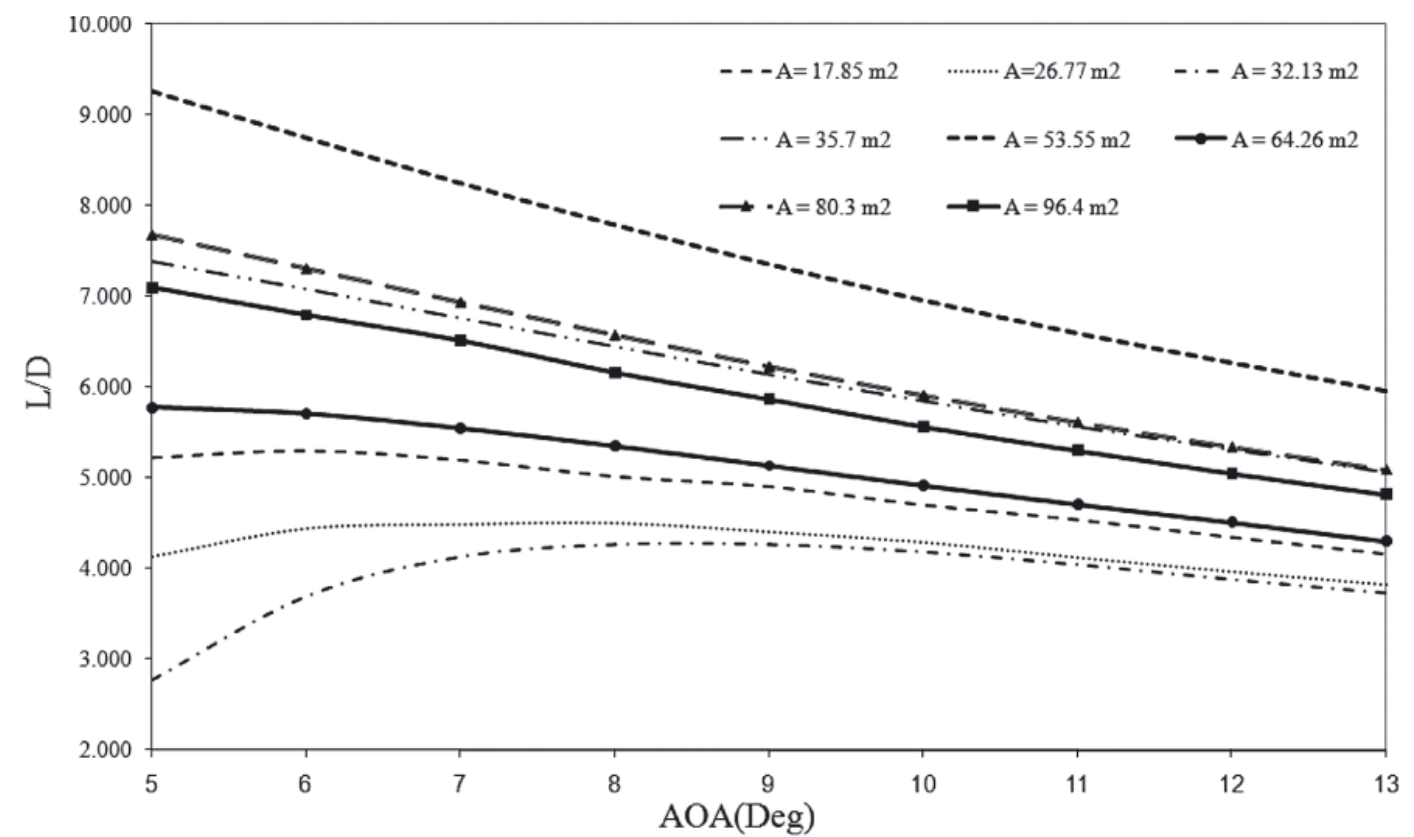

Fig. 10. The lift-to-drag ratio of air wing with respect to the angle of attack and wing area at speed of 100 knot

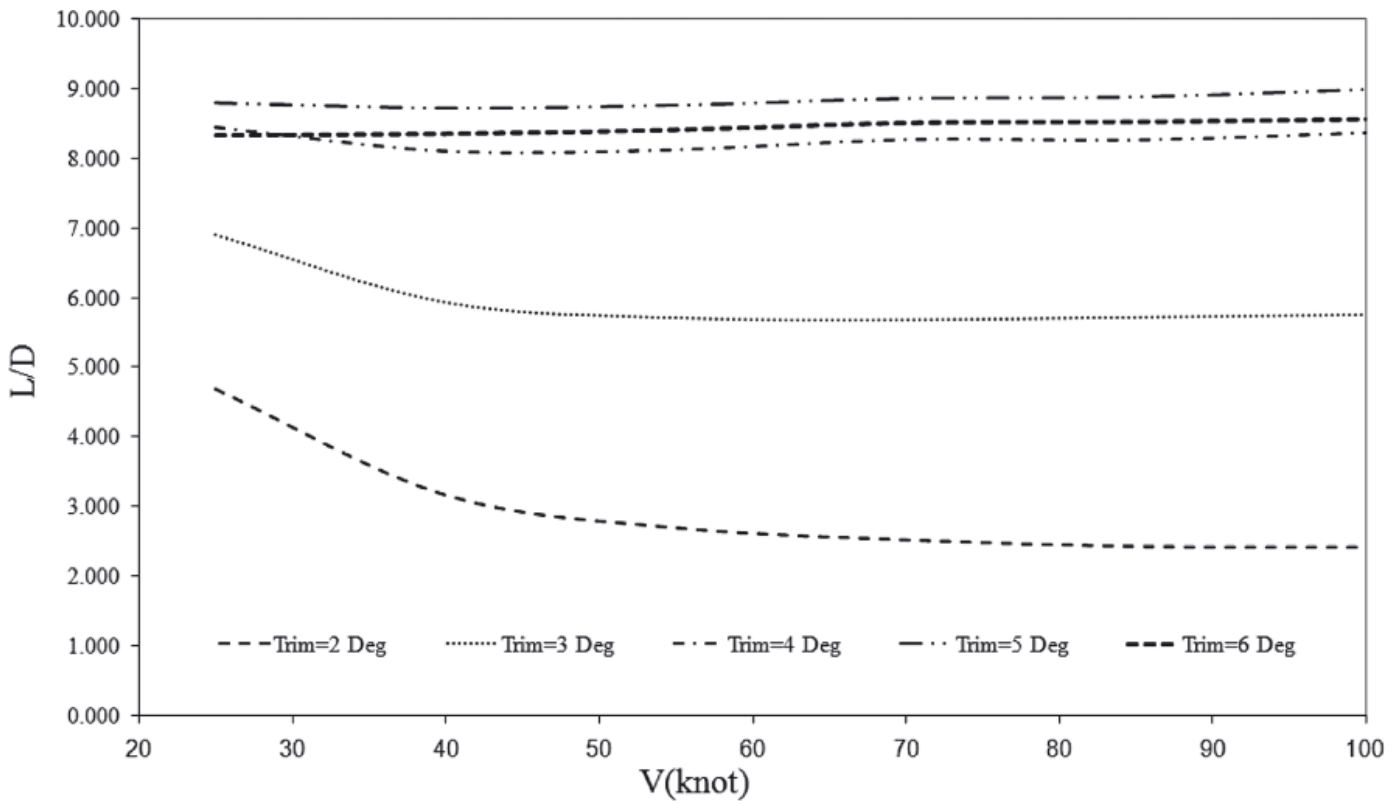

Fig. 11. The lift-to-drag ratio of a planing hull with respect to speed and trim at the draught of $0.5 \mathrm{~m}$

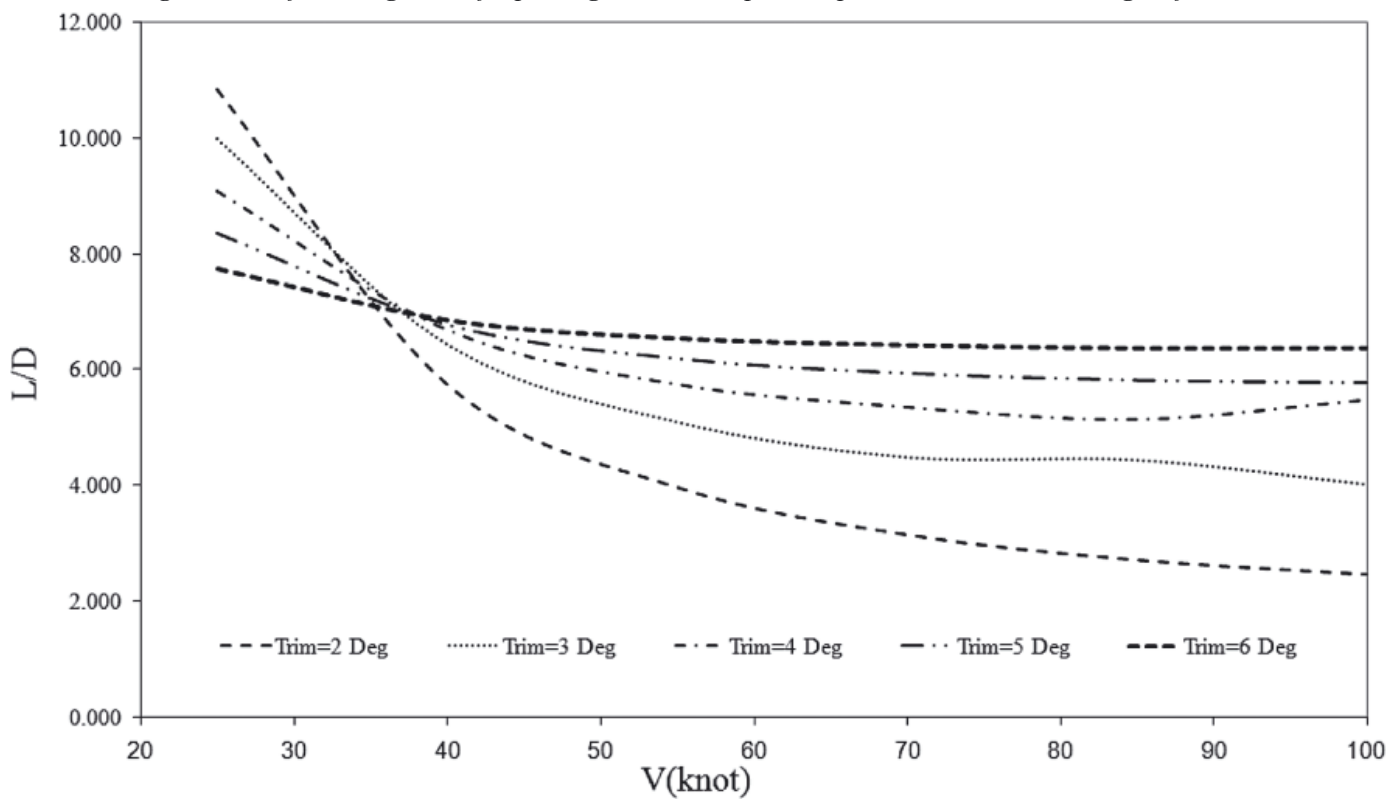

Fig. 12. The lift-to-drag ratio of a planing hull with respect to speed and trim at the draught of $0.95 \mathrm{~m}$ 


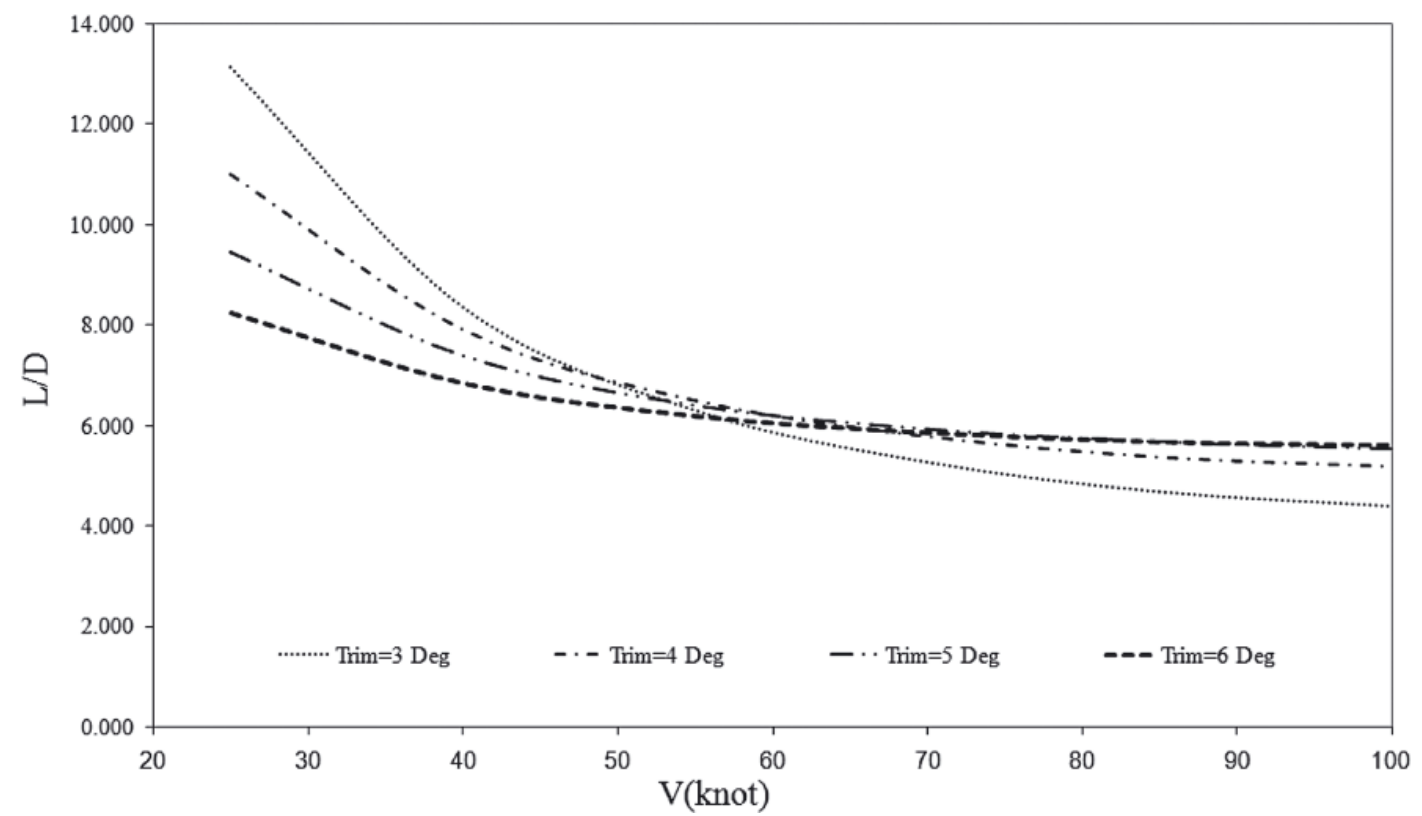

Fig. 13. The lift-to-drag ratio of a planning hull with respect to speed and trim at the draught of $1.36 \mathrm{~m}$
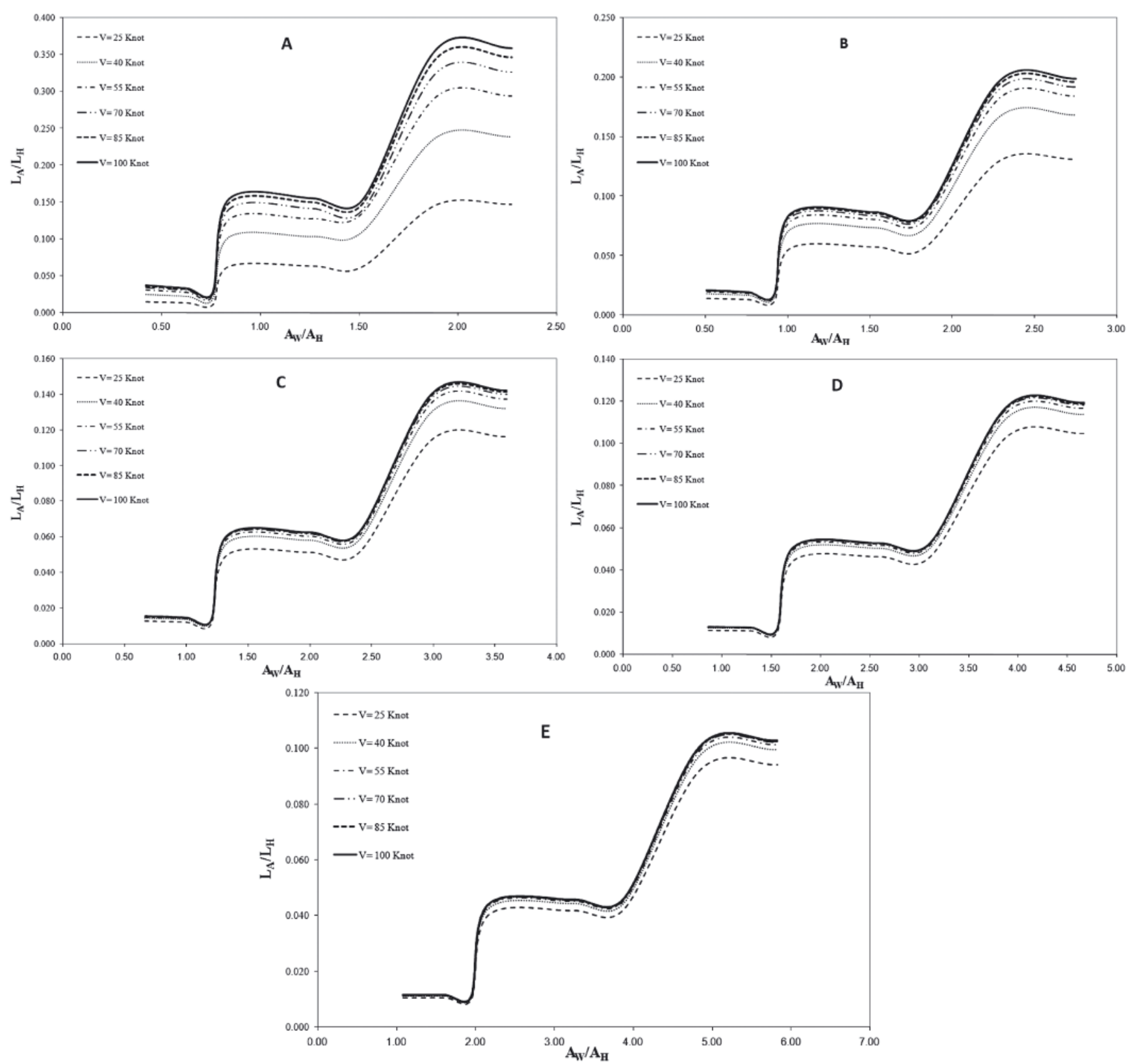

Fig. 14. The lift ratio versus the surface ratio for different speeds at the draught of $0.5 \mathrm{~m}$ 
obtains its peak value in the case of the wing of $53 \mathrm{~m}^{2}$ area, whose chord length is the least (of 50 percent of craft length) and span is the largest (of 150 percent of craft breadth). On this basis, it is concluded that the wing should be so designed as to obtain the possible highest aspect ratio.

The lift-to-drag ratio of a typical planning hull is shown in Fig. 11 through 13, which varies from 2 to 12 . It decreases as the draught and speed of the craft increases. Additionally, the greater trim the greater lift-to-drag ratio.

A comparison of the two groups of curves leads to an important conclusion that there is no certain advantage from using the wing aerodynamic force instead of the hydrodynamic lifting surface force.

\section{Comparison of hydrodynamic lift with aerodynamic lift}

The ratio of aerodynamic lift (of wings) and hydrodynamic lift (of planing hull), hereafter called the lift ratio, versus the ratio of wing area and planing wetted surface, hereafter called the surface ratio, is shown in Fig. 14.

As trim angle increases the lift ratio increases too. This is due to the positive angle of attack of 7 degree of the wings for the craft on even keel. That is why the maximum lift ratio occurs at the trim angle of $2 \mathrm{deg}$ at the speed of 100 knot. However, it may not be greater than 40 percent.

As far as the surface ratio has been concerned, increasing the surface ratio leads to increasing the lift ratio. Furthermore, the lift ratio has three distinct zones not associated with different trim angles. In the case of a low surface ratio, less than 1.0, the hydrodynamic forces plays a dominant role in supporting the craft weight while the aerodynamic force is negligible. For the surface ratio of a moderate value from the range of 1.5 up to 2.0 , the lift ratio is less than 10 percent. For the surface ratio of more than 2.0 the lift ratio may reach as much as 35 percent.

(A: trim angle of $2 \mathrm{deg}, \mathrm{B}$ : trim angle of $3 \mathrm{deg}, \mathrm{C}$ : trim angle of $4 \mathrm{deg}, \mathrm{D}$ : trim angle of $5 \mathrm{deg}$ and E: trim angle of $6 \mathrm{deg}$ )

\section{CONCLUSIONS}

A typical hybrid craft was analyzed to examine the conditions in which aerodynamic lift force of wing is beneficial for planing mono-hull. A typical planing body fitted with wings of different dimensions proportional to the main dimensions of the craft, was considered. The analysis showed that if a planingwing hybrid craft has to be used, its surface ratio should be more than two in conjunction with a high aspect ratio of wing. The main conclusions of this study are as follows:

- In the planing-wing hybrid craft the hydrodynamic lift-todrag ratio is usually slightly greater than the aerodynamic lift-to-drag ratio. Therefore, there is no comparative advantage from using air wing.

- The absolute lift force developed by the hybrid craft is a key element for supporting its weight. Generally, the maximum share of the lift force produced by the air wing is equal to about 20 percent of the hydrodynamic lift force in practical conditions.

- Some phenomena such as the hitting of spray to wings as well as impact of the wings against wave surface may produce some undesirable behaviour of a hybrid craft.

Finally, it should be mentioned that air wing may be used only in special circumstances, but not in general.

This conclusion is based on results of technical investigations only, but sometimes aesthetic view of a craft is more important than its efficiency. A planing-wing hybrid craft seems to be much more beautiful than a simple planing craft.

\section{BIBLIOGRAPHY}

1. Matveev K. I., Dubrovsky V. A.: Aerodynamic Characteristics of a Hybrid Trimaran Model. Ocean Engineering, 34(3-4),2007.

2. Shipps, P. R.: Hybrid ram-wing/planning craft - today's raceboats, tomorrow's outlook. AIAA/SNAME Advanced Marine Vehicles Conference, 1976

3. Ward, T. M., Goelzer, H. F., Cook, P. M.: Design and performance of the ram wing planning craft - KUDU II. AIAA/ SNAME Advanced Marine Vehicles Conference, 1978

4. Privalov, E. I., Kirillovikh, V. N.: Transport amphibious platforms: a new type of high-speed craft. Proceedings of Ekranoplans \& Very Fast Craft Workshop, 1996

5. Doctors, L. J.: Analysis of the efficiency of an ekranocat: a very high speed catamaran with aerodynamic alleviation. International Conference on Wing In Ground Effect Craft (WIGs '97), RINA, 1997

6. www.freepatentonline.com

7. Collu M., Patel M. H., Trarieux F.: High speed marine vehicles with aerodynamic surfaces: development of a dynamic model for a novel configuration. Cranfield Multi Strand Conference, 6-7 May 2008. Cranfield, Bedfordshire, UK

8. Collu M., Patel M. H., Trarieux F.: A Unified Mathematical Model for High Speed Hybrid (Air and Waterborne)Vehicles., $2^{\text {nd }}$ International Conference on Marine Research and Transportation, 2007

9. Collu M., Patel M. H., Trarieux F.:A Mathematical Model to analyze the Static Stability of Hybrid (Aero hydrodynamically supported) vehicles. $8^{\text {th }}$ Symposium on High Speed Marine Vehicles 2008 (HSMV08), Naples, Italy, 2008

10.www.seaphantom.com

11.Korneev, N.V., Taranov, A.E., Treshkov, V.K.: Efficient software for numerical calculation of ekranoplans (WIG craft) and hydrofoils. Proc. Workshop WISE up to Ekranoplan GEMS,The University of New South Wales, Sydney, Australia, pp. 263-266, 1998.

12.Bakhshandehrostamai A. Ghasemi H.: Design and Analysis of 2D Wing In Ground Effect Based on Maximum Lift-Drag Ratio.,MSc thesis, Amirkabir University of Technology, Tehran, Iran, 2008

13.Matjasic K. at al.: WIG (Wing In Ground effect) craft in military and paramilitary applications. NATO Conference, TO-AVT Symposium, April 7 -11, 2003

14.Park K. and Lee J.: Influence of endplate on aerodynamic characteristics of low-aspect-ratio wing in ground effect. Journal of Mechanical Science and Technology, Vol.22, 2008, pp. 2578 2589,

15.Husa B.: WIG configuration development from component matrix. Orion technology, Aerospace design and engineering, Internal report, 2000

16. Yun L. Bliault A., Doo J.: WIG craft and Ekranoplan; Ground effect craft technology. ISBN 978-1-4419-0041-8 e-ISBN 9781-4419-0042-5, Springer, 2010

\section{CONTACT WITH THE AUTHORS}

Hamid Zeraatgar, Assistant Professor

Ali Bakhshandeh Rostami, MSc.

Abolfazl Nazari, MSc.

Faculty of Marine Technology,

Amirkabir University of Technology, Somayyeh Str., Hafez Ave.

Tehran, Iran

POBox: 5875-4413

e-mail: hamidz@aut.ac.ir 